\title{
Density functional theory: a tale of success in three codes
}

\author{
D. R. Bowler \\ Dept. of Physics \& Astronomy, UCL, Gower St, London, WC1E 6BT. \\ London Centre for Nanotechnology, 19 Gordon St, London WC1H 0AH. \\ WPI-MANA, NIMS, 1-1 Namiki, Tsukuba, Ibaraki 305-0047, JAPAN. \\ E-mail: david.bowler@ucl.ac.uk \\ E-mail: @MillionAtomMan
}

This Viewpoint relates to three articles by 1) José M Soler et al (2002 J. Phys.: Condens. Matter. 14 2745), 2) M D Segall et al (2002 J. Phys.: Condens. Matter. 14 2717), 3) Paolo Giannozzi et al (2009 J. Phys.: Condens. Matter. 21 395502), and was published as part of a series of Viewpoints celebrating 50 of the most influential papers published in the Journal of Physics series, which is celebrating its 50th anniversary.

In 1929, Paul Dirac famously wrote, "The underlying physical laws necessary for the mathematical theory of a large part of physics and the whole of chemistry are thus completely known, and the difficulty is only that the exact application of these laws leads to equations much too complicated to be soluble. It therefore becomes desirable that approximate practical methods of applying quantum mechanics should be developed, which can lead to an explanation of the main features of complex atomic systems without too much computation." [1]. The papers discussed in this commentary[2, 3, 4] all describe implementations of Density Functional Theory (DFT) [5, 6] which go a considerable way to fulfillng Dirac's desireł. DFT calculations now routinely feature in physics, chemistry, materials science, earth sciences, biochemistry and engineering, both interpreting existing results and predicting experimentally inaccessible properties.

While the foundational work of DFT was published in the 1960s, the wide adoption of the method had to wait for two key developments: first, sufficient computational power to be available; second, a computationally tractable implementation. These were both fulfilled in the 1980s, with the implementation exemplified in the seminal paper by Car and Parrinello[7], which introduced plane-wave, pseudopotential DFT and enabled accurate calculations on periodic systems. The introduction of pseudopotentials, where the full nuclear potential is screened by the core electrons of the atom, and only valence electrons are considered, significantly reduced the number of states which needed to be found, as well removing the computationally expensive high kinetic energy parts of the valence wavefunctions. Plane waves, while in many ways a sub-optimal choice of basis

$\ddagger$ It is interesting to note that Dirac himself added the term for exchange to Thomas-Fermi theory, a precursor of DFT which depends only on the electron density. 
function for electrons strongly bound to nuclei, have the over-riding advantages of simple computational implementation and systematic convergence with a single parameter. The Car-Parrinello paper also introduced ab initio molecular dynamics, which is in extensive use, giving access to dynamical properties of materials. These approaches, along with a detailed discussion of various technical matters, are covered in an extensive early review[8], while a good general introduction to DFT simulation of materials is given by the CASTEP paper[2].

The CASTEP[2] and QuantumEspresso[3] codes are both periodic, plane wave pseudopotential DFT codes§, though they take different approaches to distribution of the code. CASTEP is marketed commercially, but is freely available to UK-based researchers, with the income used to support a variety of research projects. It was the first widely available DFT code, used by non-specialists. QuantumEspresso is made freely available, under the GNU General Public Licence, and its success clearly demonstrates the continuing interest in and demand for DFT codes and their continued development.

The SIESTA[4] code, which is also freely available, uses a different basis set, based around numerical atomic orbitals found using pseudopotentials (hence known as pseudo-atomic orbitals, or PAOs). This type of basis function gives a more intuitive picture of electronic structure, though cannot be systematically improved; a de facto standard where two radial functions are used for the valence orbitals, and a single polarisation function is included (giving the name double-zeta or double-numerical plus polarisation, or DZP/DNP), has emerged. Local orbital approaches give significant savings in calculating and storing the Hamiltonian and overlap matrices, and in the number of basis functions required per atom. The SIESTA code is very successful, and opened the door to simple, computationally cheap DFT calculations without the need for high-performance computing.

Local orbital methods also open the way to large-scale DFT simulations. Over the last twenty to thirty years, with the development and availablility of high-performance computing centres, it has become routine to perform calculations on hundreds of atoms, and possible to work on one or two thousand atoms. This size has not increased significantly, as standard DFT implementations scale poorly with system size (the computer effort increases with the cube of the number of atoms, while the memory required increases with the square). Recent work has given methods that scale linearly with system size[9], and simulations of over 1,000,000 atoms have been shown to be possible. There is also considerable effort to improve the accuracy of DFT, since it is well-known that it has many inaccuracies, even though the original Kohn-Sham reformulation is exact in principle.

It is important to acknowledge the vital role that software engineering plays in modern science. Both CASTEP and QuantumESPRESSO set out to follow modern principles of software engineering, adhering to modular design (and, in the case of the

$\S$ We should note that there are many of this kind of code, such as VASP, CPMD, Abinit, and no endorsement of any particular code is intended. 
present form of CASTEP, producing a complete code specification before writing any code at all). This attitude is extremely valuable, as it enables reliable code maintenance, regular testing and extension of the code to include new functionality as well as being implemented on new computational architectures (such as general-purpose GPUs).

These papers are deservedly highly cited, underlining the central position of DFT in atomistic modelling of materials of all kinds. Over the last thirty years, computational modelling has become the third leg of scientific enquiry, alongside experiments and theory. This is shown in no field more than those where DFT is in common use.

\section{Acknowledgments}

DRB has learnt about DFT from many different people, and cannot name them all here. He is especially grateful to Mike Gillan, Chris Goringe, and Tsuyoshi Miyazaki.

[1] Dirac P A M 1929 Proc. R. Soc. A 123714

[2] Segall M D, Lindan P J D, Probert M J, Pickard C J, Hasnip P J, Clark S J, and Payne M C 2002 J. Phys.: Condens. Matter 142717

[3] Giannozzi P, Baroni S, Bonini N, Calandra M, Car R, Cavazzoni C, Ceresoli D, Chiarotti G L, Cococcioni M, Dabo I, Corso A D, de Gironcoli S, Fabris S, Fratesi G, Gebauer R, Gerstmann U, Gougoussis C, Kokalj A, Lazzeri M, Martin-Samos L, Marzari N, Mauri F, Mazzarello R, Paolini S, Pasquarello A, Paulatto L, Sbraccia C, Scandolo S, Sclauzero G, Seitsonen A P, Smogunov A, Umari P, and Wentzcovitch R M 2009 J. Phys.: Condens. Matter 21395502

[4] Soler J M, Artacho E, Gale J D, Garca A, Junquera J, n P O, and nchez Portal D S 2002 J. Phys.: Condens. Matter 142745

[5] Hohenberg P and Kohn W 1964 Phys. Rev. 136 B864

[6] Kohn W and Sham L J 1965 Phys. Rev. 140 A1133

[7] Car R and Parrinello M 1985 Phys. Rev. Lett. 552471

[8] Payne M C, Teter M P, Allan D C, Arias T A, and Joannopoulos J D 1992 Rev. Mod. Phys. 64 1045

[9] Bowler D R and Miyazaki T 2012 Rep. Prog. Phys. 7536503 